\title{
The existence of solutions for general variational inequality and applications in FC spaces
}

Jinghai Wang

Correspondence: fzwjh@163.com Institute of Mathematics and Computer Science, Fuzhou University, Fuzhou, Fujian 350002, P. R China

\begin{abstract}
In this article, we prove the existence of solutions for the general variational inequality $\varphi(x, y) \geq f(x)-f(y)$ and Minty type theorem by using the generalized KKM theorem in topological spaces without linear structure. Some properties of solutions set for the general variational inequality are studied by Minty type theorem. As applications, equivalence between Browder fixed point theorem and Ky Fan's minimax inequality are studied in topological spaces.
\end{abstract}

Keywords: generalized R-KKM mapping, general variational inequality, minty type theorem, fixed point

\section{Introduction}

The existence of solutions and the properties of solutions set for general variational inequality were studied by many authors in linear topologcal spaces (see [1-7]). These results depend deeply on the linear structure of spaces. In 1987, Horvath [8] used the concept of retractile subset family to replace the convexity assumptions and established the KKM theorem in $H$-space. Motivated by Horvath's work, the various extensions of KKM theorem and their applications in many aspects were investigated by various authors under the assumption without convexity. Recently, Ding [9,10] and Deng and Xia [11] introduced the class of finitely continuous topological spaces (in short FCspaces) without any convexity and linear structure. The class of $F C$-spaces includes the classes of $H$-spaces, $G$-convex spaces, $G$ - $H$-convex spaces, $L$-convex spaces and many topological spaces with convexity structure. The purpose of this article is to study the existence of solutions for the following general variational inequality (in short VI(1.1))

$$
\phi(x, y) \geq f(x)-f(y)
$$

Minty type theorem and the properties of the solutions set for general variational inequality(1.1) in $F C$-spaces.

\section{Preliminaries}

Let $E$ be a topological space, $X$ be a nonempty subset of $E$. Throughout this article, we assume that $\langle X\rangle$ denotes all nonempty finite subset of $X, \Delta_{n}$ denotes the standard $n$ simplex $\left(e_{0}, \ldots, e_{\mathrm{n}}\right)$ in $R^{n+1}, \Delta_{k}$ denotes $k$-sub-simplex of $\Delta_{n}$ with vectors $\left(e_{i_{0}}, \ldots, e_{i_{k}}\right)$, $(0 \leq k \leq n), R=(-\infty,+\infty), \bar{R}=(-\infty,+\infty)$. The following definitions can be found in $[4,7,9,11]$.

(C) 2012 Wang; licensee Springer. This is an open access article distributed under the terms of the Creative Commons Attribution License (http://creativecommons.org/licenses/by/2.0), which permits unrestricted use, distribution, and reproduction in any medium, provided the original work is properly cited. 
Definition 2.1. $\left(E, \phi_{N}\right)$ is said to be a finitely continuous space (in short $F C$-space) if $E$ is a topological space and for each $N=\left\{x_{0}, \ldots, x_{n}\right\}\lfloor\langle E\rangle$, there exists a continuous mapping $\phi_{N}: \Delta_{n} \rightarrow E$. A subset $X$ of $\left(E, \phi_{N}\right)$ is said to be an $F C$-subspace of $E$ if for each $N=\left\{x_{0}, \ldots, x_{n}\right\}\left\lfloor\langle E\rangle\right.$ and for any $\left\{x_{i_{0}}, \ldots, x_{i_{k}}\right\} \subset X \cap N, \phi_{N}\left(\Delta_{k}\right) \subset X$, where $\Delta_{k}=\operatorname{co}\left\{e_{i_{j}}: j=0, \ldots, k\right\}$.

Definition 2.2. Let $X$ be an $F C$-subspace of $\left(E, \phi_{N}\right)$.

(1) $f: X \rightarrow R$ is said to be an R-convex function if for each $N=\left\{x_{0}, \ldots, x_{n}\right\} \in\langle X\rangle$, $f\left(x_{t}\right) \leq \sum_{i=0}^{n} t_{i} f\left(x_{i}\right)$, where $x_{t}=\varphi_{N}\left(\sum_{i=0}^{n} t_{i} e_{i}\right), t_{i} \in[0,1], \sum_{i=0}^{n} t_{i}=1$.

(2) $f: X \rightarrow R$ is said to be a strictly R-convex function if for each $N=\left\{x_{0}, x_{1}\right\} \in\langle X\rangle$ and $x_{0} \neq x_{1}, f\left(x_{t}\right)<t f\left(x_{0}\right)+(1-t) f\left(x_{1}\right)$, where $x_{t}=\phi_{N}\left(t e_{0}+(1-t) e_{1}\right), t \in(0,1)$.

(3) $\phi: X \times X \rightarrow \bar{R}$ is said to be $\lambda$-quasi-convex related to the second variant if subset $\{y \in X ; \varphi(x, y)<\lambda\}$ is an FC-subspace for any $x \in X . \varphi$ is said to be R-quasi-convex related to the second variant if $\varphi$ is $\lambda$-quasi-convex related to the second variant for any $x \in X$ and $\lambda \in R$.

(4) $\phi: X \times X \rightarrow \bar{R}$ is said to be an R-semi-continuous function if for any $N=\left\{x_{0}, x_{1}\right\} \in$ $\langle X\rangle$,

$$
\varlimsup_{t \rightarrow 0^{+}} \phi\left(x_{t}, x_{1}\right) \leq \phi\left(x_{0}, x_{1}\right),
$$

where $x_{t}=\phi_{N}\left((1-t) e_{0}+t e_{1}\right), t \in[0,1]$.

Definition 2.3. Let $X$ be a nonempty subset of $E$. A function $\phi: X \times X \rightarrow \bar{R}$ is said to be monotone if $\varphi(x, y)+\varphi(y, x) \leq 0$ for any $x, y \in X$. In addition, if $\varphi$ also satisfies the condition $\varphi(x, y)+\varphi(y, x)=0$ if and only if $x=y$, then $\varphi$ is said to be strictly monotone.

Definition 2.4. Let $X$ be a nonempty set and $\left(Y, \phi_{N}\right)$ be an $F C$-space. For $\lambda \in R$, a function $\phi: X \times Y \rightarrow \bar{R}$ is said to be $\lambda$-generalized R-diagonally quasi-convex (resp., $\mathrm{R}$-diagonally quasi-concave) related to the first variant if, for each $\left\{x_{0}, \ldots, x_{n}\right\} \in\langle X\rangle$, there exists $N=\left\{y_{0}, \ldots, y_{n}\right\}\left\lfloor\langle Y\rangle\right.$ such that, for each subset $\left\{i_{0}, \ldots, i_{k}\right\} \subset\{0, \ldots, n\}$ and $\max _{0 \leq j \leq k} \phi\left(x_{i^{\prime}}, \bar{y}\right) \geq \lambda, \quad\left(\right.$ resp. $\left.\min _{0 \leq j \leq k} \phi\left(x_{i_{j}}, \bar{y}\right) \leq \lambda\right)$,

$$
\max _{0 \leq j \leq k} \phi\left(x_{i j}, \bar{y}\right) \geq \lambda, \quad\left(\text { resp. } \min _{0 \leq j \leq k} \phi\left(x_{i j}, \bar{y}\right) \leq \lambda\right) .
$$

where $\Delta_{k}$ denotes $k$-sub-simplex of with vectors $\left(e_{i_{0}}, \ldots, e_{i_{k}}\right),(0 \leq k \leq n)$ related to $\left\{\gamma_{i_{0}}, \ldots, \gamma_{i_{k}}\right\}$.

Definition 2.5. Let $X$ be a nonempty set and $\left(Y, \phi_{N}\right)$ be an $F C$-space. A mapping $T$ : $X \rightarrow 2^{Y}$ is said to be a generalized R-KKM mapping if, for each finite subset $\left\{x_{0}, \ldots, x_{n}\right\}$ $\in\langle X\rangle$, there exists $N=\left\{y_{0}, \ldots, y_{n}\right\} \in\langle Y\rangle$ such that, for each subset $\left\{i_{0}, \ldots, i_{k}\right\} \subset\{0, \ldots, n\}$, $\varphi_{N}\left(\Delta_{k}\right) \subset \cup_{j=0}^{k} T\left(x_{i_{j}}\right)$.

Lemma 2.1. $\varphi$ is $\lambda$-generalized R-diagonally quasi-convex (resp., R-diagonally quasiconcave) related to the first variant if and only if the mapping $G: X \rightarrow 2^{Y}$ defined by

$$
G(x)=\{y \in Y ; \phi(x, y) \geq \lambda\},(\text { resp. } G(x)=\{y \in Y ; \phi(x, y) \leq \lambda\}
$$

is a generalized R-KKM mapping.

Proof If $\varphi$ is $\lambda$-generalized R-diagonally quasi-convex related to the first variant, then for each $\left\{x_{0}, \ldots, x_{n}\right\} \in\langle X\rangle$, there exists $N=\left\{y_{0}, \ldots, y_{n}\right\} \in\langle Y\rangle$ such that, for each subset 
$\left\{i_{0}, \ldots, i_{k}\right\} \in\{0, \ldots, n\}$ and $\bar{y} \in \varphi_{N}\left(\Delta_{k}\right), \max _{0 \leq j \leq k} \phi\left(x_{i_{j}}, \bar{y}\right) \geq \lambda$. Hence, there exists some $j_{0} \in$ $\{0, \ldots, k\}$ such that $\phi\left(x_{i_{j_{0}}}, \bar{y}\right) \geq \lambda$, i.e., $\bar{\gamma} \in G\left(x_{i_{j_{0}}}\right)$, therefore $\varphi_{N}\left(\Delta_{k}\right) \subset \cup_{j=0}^{k} G\left(x_{i_{j}}\right)$, i.e., $G$ is a generalized R-KKM mapping.

Conversely, suppose that $G$ is a generalized R-KKM mapping. Then for each $\left\{x_{0}, \ldots, x_{n}\right\}$ $\in\langle X\rangle$, there exists $N=\left\{y_{0}, \ldots, y_{n}\right\} \in\langle Y\rangle$ such that, for each subset $\left\{i_{0}, \ldots, i_{k}\right\} \subset\{0, \ldots, n\}$, $\varphi_{N}\left(\Delta_{k}\right) \subset \cup_{j=0}^{k} G\left(x_{i j}\right)$. Thus for any $\bar{y} \in \varphi_{N}\left(\Delta_{k}\right)$, there exists some $j_{0} \in\{0, \ldots, k\}$ such that $\bar{y} \in G\left(x_{i_{j_{0}}}\right)$, i.e., $\phi\left(x_{i_{j_{0}}}, \bar{y}\right) \geq \lambda$. It follows that $\max _{0 \leq j \leq k} \phi\left(x_{i_{j}}, \bar{y}\right) \geq \lambda$. This completes the proof.

Definition 2.6. Let $X, Y$ be two nonempty subsets of $E$. For $\lambda \in R$, a function $\phi: X \times Y \rightarrow \bar{R}$ is said to be $\lambda$-transfer compactly lower (resp., upper) semi-continuous relate to the second variant if, for each nonempty compact subset $K \subset Y$ and each $y \in$ $K$, there exists $x \in X$ such that $\varphi(x, y)>\lambda$ (resp., $\varphi(x, y)<\lambda$ ) implies that there exists an open neighborhood $U(y)$ of $y$ in $K$ and a point $\bar{x} \in X$ such that $\phi(\bar{x}, z)>\lambda$ (resp., $\phi(\bar{x}, y)<\lambda)$ for all $z \in U(y)$.

Definition 2.7. Let $X, Y$ be two nonempty subsets of $E$. A mapping $T: X \rightarrow 2^{Y}$ is said to be transfer compactly closed valued on $X$ if, for $x \in X$ and for each nonempty compact subset $K$ of $Y, y \notin T(x) \cap K$ implies that there exists a point $\bar{x} \in X$ such that $y \notin c l_{K}(T(\bar{x}) \cap K)$, where $c l_{K}(T(\bar{x}) \cap K)$ is the closure of $T(\bar{x}) \cap K$ in $K$.

Lemma 2.2. Let $T: X \rightarrow 2^{Y}$ be a set-valued mapping defined by

$$
T(x)=\{y \in Y ; \phi(x, y) \leq \lambda\},(\text { resp. } T(x)=\{y \in Y ; \phi(x, y) \geq \lambda\}) .
$$

Then $T$ is transfer compactly closed valued if and only if $\varphi$ is $\lambda$-transfer compactly lower (resp., upper) semi-continuous related to second variant.

Proof. Suppose that $T$ is transfer compactly closed valued. If for each nonempty compact subset $K \subset Y$ and each $y \in K$, there exists $x \in X$ such that $\varphi(x, y)>\lambda$ (resp., $\phi(\bar{x}, y)<\lambda)$, then $y \notin T(x) \cap K$. Thus, there exists a point $\bar{x} \in X$ such that $y \notin c l_{K}(T(\bar{x}) \cap K)$. Hence, there exists an open neighborhood $U(y)$ of $y$ in $K$ such that $U(y) \cap T(\bar{x})=\emptyset$, i.e., $\phi(\bar{x}, z)>\lambda$ (resp., $\phi(\bar{x}, z)<\lambda$ ) for all $z \in U(y)$.

Conversely, for each nonempty compact subset $K \subset Y$ and for any $y \notin T(x) \cap K$, we have (i) if $y \notin K$ then $y \notin c l_{K}(T(\bar{x}) \cap K$ ); (ii) if $y \in K$ and $y \notin T(x)$ then $\varphi(x, y)>\lambda$ (resp., $\phi(\bar{x}, z)<\lambda)$. Hence, there exists an open neighborhood $U(y)$ of $y$ in $K$ and $\bar{x} \in X$ such that $\phi(\bar{x}, z)>\lambda$ (resp. $\phi(\bar{x}, z)<\lambda$ ) for all $z \in U(y)$, therefore $y \notin c l_{K}(T \bar{x})$. This implies that $T$ is transfer compactly closed valued. The proof is completed.

\section{Solutions of $\mathrm{VI}(1.1)$}

In the Sections 3 and 4 , we assume that $\left(E, \phi_{N}\right)$ is an $F C$-space, $X$ is a nonempty $F C$ -

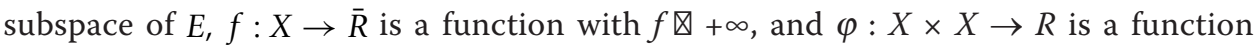
with $\varphi(x, x) \geq 0$ for all $x \in X$.

Theorem 3.1. Suppose that

(i) there exists a compact subset $K$ of $E$ and $x^{*} \in X \cap K$ such that

$$
f(x)>\phi\left(x, x^{*}\right)+f\left(x^{*}\right) \quad(\forall x \in X \backslash K) ;
$$

(ii) $f(y)+\varphi(x, y)-f(x)$ is 0 -generalized R-diagonally quasi-convex related to the variant $y$;

(iii) $f(x)-\varphi(x, y)-f(y)$ is 0 -transfer compactly lower semi-continuous related to the variant $x$. 
Then VI(1.1) has a solution in $X \cap K$, i.e., there exists $\bar{x} \in X \cap K$ such that

$$
\phi(\bar{x}, y) \geq f(\bar{x})-f(y) \quad(\forall y \in X) .
$$

Proof. For any $y \in X$, let

$$
G(y)=\{x \in X ; f(y)+\phi(x, y) \geq f(x)\}
$$

Then $G$ is a multi-valued mapping from $X$ into itself. It is easy to see that, if $\bar{x} \in \cap_{y \in X} G(\gamma) \neq \varnothing$, then $\bar{x}$ is a solution of $\mathrm{VI}(1.1)$.

We now show that $\cap_{y \in X} G(\gamma) \neq \emptyset$. In fact, condition (ii) and Lemma 2.1 imply that $G$ is a generalized R-KKM mapping, condition (iii) and Lemma 2.2 imply that $G$ is transfer compactly closed valued. It follows from condition (i) that $x \notin G\left(x^{*}\right)$ for all $x \in X$ $\backslash K$, and so $G\left(x^{*}\right) \subset K$, i.e., $\overline{G\left(x^{*}\right)}$ is a compact subset of $E$. By Theorem 3.4 in [11], $\cap_{y \in X} G(\gamma) \neq \emptyset$. In addition, $\cap_{y \in X} G(\gamma) \subset G\left(x^{*}\right) \subset K$, and so the solutions of VI(1.1) is in $X \cap K$. This completes the proof.

Theorem 3.2. Suppose that

(i) there exist a compact subset $K$ of $E$ and $x^{*} \in X \cap K$ such that

$$
f(x)>\phi\left(x, x^{*}\right)+f\left(x^{*}\right) \quad\left(\forall x^{*} \in X \backslash K\right) ;
$$

(ii) $f(y)+\varphi(x, y)$ is R-quasi-convex related to the variant $y$;

(iii) $f(x)-\varphi(x, y)$ is lower semi-continuous related to the variant $x$.

Then, $\mathrm{VI}(1.1)$ has a solution in $X \cap K$.

Proof. We first show the multi-valued mapping $G$ defined by (3.1) is a generalized RKKM mapping. Suppose that $G$ is not a generalized R-KKM mapping, then there exist $N=\left\{x_{0}, \ldots, x_{n}\right\} \in\langle X\rangle,\left\{e_{i_{0}}, \ldots, e_{i_{k}}\right\} \subset\left\{e_{0}, \ldots, e_{n}\right\}$ and $y^{\prime} \in \phi_{N}\left(\Delta_{k}\right)$ such that

$y^{\prime} \notin \bigcup_{j=0}^{k} G\left(x_{i_{j}}\right)$. Since $f(x)+\varphi(x, y)$ is R-quasi-convex related to the variant $y$ and $\left\{x_{i_{0}}, \ldots, x_{i_{k}}\right\} \subset\left\{y \in X: f(y)+\varphi\left(y^{\prime}, y\right)<f\left(y^{\prime}\right)\right\}$, then $\phi_{N}\left(\Delta_{k}\right) \subset\left\{y \in X: f(y)+\phi\left(y^{\prime}, y\right)<f\right.$ $\left.\left(y^{\prime}\right)\right\}$. And $y^{\prime} \in \phi_{N}\left(\Delta_{k}\right)$, then $f\left(y^{\prime}\right)+\phi\left(y^{\prime}, y^{\prime}\right)<f\left(y^{\prime}\right)$, i.e., $\phi\left(y^{\prime}, y^{\prime}\right)<0$, this contracts $\phi\left(y^{\prime}, y^{\prime}\right)$ $\geq 0$. Therefore, $G$ is a generalized R-KKM mapping.

Condition (i) implies that $x \notin G\left(x^{*}\right)$ for all $x \in X \backslash K$, so, $G\left(x^{*}\right) \subset K$, i.e., $\overline{G\left(x^{*}\right)}$ is a compact subset of $E$. It follows from condition (iii) that $G(y)$ is a closed subset for each $y \in X$. By Theorem 3.2 in [11], $\cap_{y \in X} G(y) \neq \varnothing$, and so $\operatorname{VI}(1.1)$ has a solution in $X \cap$ $K$. This completes the proof.

By Theorems 3.1 and 3.2, it is easy to get Ky Fan's minimax inequality in FC-spaces.

Corollary 3.1. Assume that $\left(E, \phi_{N}\right), X, f, \varphi$ satisfy the conditions (ii) and (iii) in Theorem 3.1 .

Then there exists $\bar{x} \in X$ such that

$$
f(y)+\phi(\bar{x}, y) \geq f(\bar{x}) \quad(\forall y \in X) .
$$

Corollary 3.2 Assume that $\left(E, \phi_{N}\right), X, f, \varphi$ satisfy the conditions (ii) and (iii) in Theorem 3.2 .

Then there exists $\bar{x} \in X$ such that

$$
f(y)+\phi(\bar{x}, y) \geq f(\bar{x}) \quad(\forall y \in X) .
$$

Remark 3.1 Theorems 3.1 and 3.2, Corollaries 3.1 and 3.2 extend some results of Gwinner [1] and Ky Fan minimax inequality to $F C$-spaces without linear structure. 


\section{Minty theorem and monotone variational inequality}

In this section, we present Minty type theorem in $F C$-spaces.

Theorem 4.1. Suppose that

(i) $\varphi: X \times X \rightarrow R$ is a monotone and $\mathrm{R}$-semi-continuous mapping;

(ii) for each $x \in X, f(y)+\varphi(x, y)$ is an R-convex function related to the variant $y$;

(iii) for any $N=\left\{x_{0}, x_{1}\right\} \in\langle X\rangle$, $\liminf _{t \rightarrow 0^{+}} f\left(x_{t}\right) \geq f\left(x_{0}\right)$, where $x_{t}=\phi_{N}\left((1-t) e_{0}+t e_{1}\right), t \in$ $[0,1]$.

Then there exists $\bar{x} \in X$ such that $f(y)+\phi(\bar{x}, y) \geq f(\bar{x})$ for all $y \in X$ if and only if $f(y)-\phi(y, \bar{x}) \geq f(\bar{x})$ for all $y \in X$.

Proof. For each $y \in X$, let

$$
\begin{aligned}
& M(y)=\{x \in X ; f(y)+\phi(x, y)-f(x) \geq 0\}, \\
& N(y)=\{x \in X ; f(y)-\phi(y, x)-f(x) \geq 0\} .
\end{aligned}
$$

It is sufficient to prove that

$$
\bigcap_{y \in X} M(\gamma)=\bigcap_{y \in X} N(y) .
$$

Since $\varphi$ is monotone, it is easy to see that $\cap_{y \in X} M(\gamma) \subset \cap_{y \in X} N(\gamma)$. We claim that

$$
\bigcap_{y \in X} N(y) \subset \bigcap_{y \in X} M(y)
$$

Suppose that (4.4) is not true. Then there exists $\bar{x} \in \cap_{y \in X} N(\gamma)$ and $\bar{x} \notin \cap_{y \in X} M(y)$ i.e.,

$$
f(\bar{x})+\phi(y, \bar{x}) \leq f(y) \quad(\forall y \in X),
$$

and there exists $\bar{\gamma} \in X$ such that

$$
f(\bar{y})+\phi(\bar{x}, \bar{y})<f(\bar{x}) .
$$

Let $A=\{\bar{x}, \bar{y}\}$, Setting $x_{t}=\phi_{A}\left((1-t) e_{0}+t e_{1}\right)$ for each $t \in[0,1]$. By condition (iii), we have

$$
\liminf _{t \rightarrow 0^{+}} f\left(x_{t}\right) \geq f(\bar{x}) .
$$

Since $\varphi$ is R-semi-continuous, $\limsup _{t \rightarrow \sigma^{+}} \phi\left(x_{t}, \bar{y}\right) \leq \phi(\bar{x}, \bar{y})$, we have

$$
\liminf _{t \rightarrow 0^{+}}\left(f\left(x_{t}\right)-\phi\left(x_{t}, \bar{y}\right)\right) \geq f(\bar{x})-\phi(\bar{x}, \bar{y})>f(\bar{y}) .
$$

Thus, there exists $t^{*} \in[0,1]$ such that

$$
f\left(x_{t}\right)-\phi\left(x_{t}, \bar{y}\right)>f(\bar{y}) \quad\left(\forall t \in\left(0, t^{*}\right]\right),
$$

therefore, we have

$$
f(\bar{\gamma})+\phi\left(x_{t}, \bar{y}\right)<f\left(x_{t}\right) \quad\left(\forall t \in\left(0, t^{*}\right]\right) .
$$

Setting $y=x_{t}$ in (4.5), we get

$$
f(\bar{x})+\phi\left(x_{t}, \bar{x}\right) \leq f\left(x_{t}\right) .
$$


Combining (4.7) and (4.8), we have

$$
(1-t)\left(f(\bar{y})+\phi\left(x_{t}, \bar{y}\right)\right)+t\left(f(\bar{x})+\phi\left(x_{t}, \bar{x}\right)\right)<f\left(x_{t}\right) .
$$

It follows from condition (ii) that

$$
(1-t)(f(\bar{y})+\phi(x, \bar{y}))+t\left(f(\bar{x})+\phi\left(x_{t}, \bar{x}\right)\right) \geq f\left(x_{t}\right)+\phi\left(x_{t}, x_{t}\right) .
$$

Combining (4.9) and (4.10), we have $\varphi\left(x_{t}, x_{t}\right)<0$, this contradicts to $\varphi\left(x_{t}, x_{t}\right) \geq 0$. Hence, (4.4) is true. The proof is completed.

Remark 4.1. Theorem 4.1 extends famous Minty theorem in $F C$-spaces without linear structure.

By using Minty type theorem (Theorem 4.1), we now discuss the properties of the solutions set for $\mathrm{VI}(1.1)$.

Theorem 4.2. Suppose that

(i) there exist a compact subset $K$ of $E$ and $x^{*} \in X \cap K$ such that

$$
f(x)>\phi\left(x, x^{*}\right)+f\left(x^{*}\right) \quad(\forall x \in X \backslash K) ;
$$

(ii) for each $x \in X, f(y)+\varphi(x, y)$ is a R-convex function related to the variant $y$;

(iii) $f(x)-\varphi(x, y)$ is lower semi-continuous related to the variant $x$;

(iv) $\varphi(x, y)$ is a monotone and R-semi-continuous mapping;

(v) for any $N=\left\{x_{0}, x_{1}\right\} \in\langle X\rangle$, $\liminf _{t \rightarrow 0^{+}} f\left(x_{t}\right) \geq f\left(x_{0}\right)$, where $x_{t}=\phi_{N}\left((1-t) e_{0}+t e_{1}\right), t \in$ $[0,1]$.

Then the solution set for $\mathrm{VI}(1.1)$ is a nonempty compact $F C$-subspace of $E$ in $X \cap K$.

Furthermore, either (I) $\varphi$ is a strictly monotone function in (iv) or (II) $f(y)+\varphi(x, y)$ is strictly R-convex function in (ii), then the solution of VI(1.1) is unique in $X \cap K$.

Proof Let $S$ be the solution set for $\mathrm{VI}(1.1)$ in $X \cap K$. It is clear that $S$ is nonempty by Theorem 3.2 and $S=\cap_{y \in X} M(\gamma)$, where $M(y)$ is defined by (4.1). We show that $S$ is a compact $F C$-subspace of $E$ in $X \cap K$. In fact, from Theorem 4.1, we have $S=\cap_{y \in X} M(\gamma)=\cap_{y \in X} N(\gamma)$, where $N(y)$ is defined by (4.2). Since $f(y)+\varphi(x, y)$ is a Rconvex function related to the variant $y$, it is easy to see that $N(y)$ is an $F C$-subspace of $E$, then $S=\cap_{y \in X} N(\gamma)$ is an $F C$-subspace of $E$. By condition (i) and (iii), we have $S \subset K$ and $S=\cap_{y \in X} M(\gamma)$ is closed. Therefore, $S$ is a nonempty compact $F C$-subspace of $E$ in $X \cap K$.

We now prove that the solution of $\mathrm{VI}(1.1)$ is unique in $X \cap K$ under the condition (I). In fact, let $x_{1}, x_{2} \in X \cap K$ be two solutions of $\mathrm{VI}(1.1)$ with $x_{1} \neq x_{2}$. Then

$$
f(y)+\phi\left(x_{1}, y\right) \geq f\left(x_{1}\right) \quad(\forall y \in X)
$$

and

$$
f(\gamma)+\phi\left(x_{2}, y\right) \geq f\left(x_{2}\right) \quad(\forall y \in X) .
$$

Putting $y=x_{2}$ in (4.11) and $y=x_{1}$ in (4.12), respectively, we have

$$
\begin{aligned}
& f\left(x_{2}\right)+\phi\left(x_{1}, x_{2}\right) \geq f\left(x_{1}\right), \\
& f\left(x_{1}\right)+\phi\left(x_{2}, x_{1}\right) \geq f\left(x_{2}\right) .
\end{aligned}
$$

Adding (4.13) and (4.14), we have $\varphi\left(x_{1}, x_{2}\right)+\varphi\left(x_{2}, x_{1}\right) \geq 0$. This implies that $\varphi\left(x_{1}, x_{2}\right)$ $+\varphi\left(x_{2}, x_{1}\right)=0$ and so $x_{1}=x_{2}$. 
Now we prove that the solution of $\mathrm{VI}(1.1)$ is unique in $X \cap K$ under condition (II). In fact, suppose that $x_{1}, x_{2} \in X \cap K$ be two solutions of $\mathrm{VI}(1.1)$ with $x_{1} \neq x_{2}$. Let $N=\left\{x_{1}\right.$, $\left.x_{2}\right\}$ and $\bar{x}=\varphi_{N}\left(\frac{1}{2} e_{0}+\frac{1}{2} e_{1}\right) \in X$. Then

$$
\frac{1}{2}\left(f\left(x_{1}\right)+f\left(x_{2}\right)+\phi\left(x_{1}, x_{2}\right)+\phi\left(x_{1}, x_{1}\right)\right)>f(\bar{x})+\phi\left(x_{1}, \bar{x}\right) \geq f\left(x_{1}\right)
$$

Therefore, we have

$$
\phi\left(x_{1}, x_{1}\right)+\phi\left(x_{1}, x_{2}\right)>f\left(x_{1}\right)-f\left(x_{2}\right) .
$$

Since $\varphi(x, y)$ is monotone, we know that $\varphi(x, y)+\varphi(y, x) \leq 0$ for any $x, y \in X$. Especially setting $x=y$, we have $\varphi(x, x) \leq 0$. Since $\varphi(x, x) \geq 0$ for all $x \in X$, it follows that $\varphi(x, x)=0$. Therefore (4.15) implies that $\varphi\left(x_{1}, x_{2}\right)>f\left(x_{1}\right)-f\left(x_{2}\right)$. Analogously, we have $\varphi$ $\left(x_{2}, x_{1}\right)>f\left(x_{2}\right)-f\left(x_{1}\right)$ and so $\varphi\left(x_{1}, x_{2}\right)+\varphi\left(x_{2}, x_{1}\right)>0$, this contradicts to the fact that $\varphi$ is monotone. Thus, the solution of $\mathrm{VI}(1.1)$ is unique. This completes the proof.

\section{Applications}

In 1968, Browder [12] established the fixed point theorem for set-valued mappings in Hausdorff topological vector spaces. This theorem plays an important role in solving quasi-variational inequality (see $[3,13]$ ). As applications, we now generalize the Browder fixed point theorem to $F C$-spaces by using Corollary 3.4 and prove the equivalent relation between Browder fixed point theorem and Ky Fan's minimax inequality in FCspaces without linear structure.

Theorem 5.1. Let $\left(E, \phi_{N}\right)$ be an $F C$-space and $X$ be a compact $F C$-subspace of $E$. Suppose that $T: X \rightarrow 2^{X}$ is a set-valued mapping satisfying one of the following conditions

(i) for each $x \in X, T(x)$ is a nonempty $F C$-subspace of $X$ and for each $y \in X, T^{-1}(y)$ is an open subset of $X$;

(ii) for each $x \in X, T(x)$ is an open subset of $X$, and for each $y \in X, T^{-1}(y)$ is a nonempty $F C$-subspace of $X$.

Then $T$ has a fixed point in $X$.

Proof. Suppose that condition (i) is satisfied and $T$ has no fixed point in $X$. For $x, y$ $\in X$, let

$$
\phi(x, y)= \begin{cases}-1, & ((x, y) \in \operatorname{graph}(T)), \\ 0, & ((x, y) \notin \operatorname{graph}(T)),\end{cases}
$$

where $\operatorname{graph}(T)=\{(x, y) ; y \in T(x)\}$. Since for each $x \in X, x \notin T(x)$ we have $\varphi(x, x)=$ 0 . Now we verify that all conditions of Corollary 3.2 are satisfied. In fact, for $\lambda \in R$ and $x \in X$, we have

$$
\{y \in X ; \phi(x, y)<\lambda\}= \begin{cases}X, & (\lambda>0) \\ T(x), & (-1<\lambda \leq 0) \\ 0, & (\lambda \leq-1)\end{cases}
$$

This implies that $\varphi(x, y)$ is $\mathrm{R}$-quasi-convex related to the variant $y$. For each $y \in X$ and $\lambda \in R$,

$$
\{x \in X ; \phi(x, y) \geq \lambda\}= \begin{cases}0, & (\lambda \geq 0), \\ X \backslash T^{-1}(y), & (-1<\lambda \leq 0), \\ X, & (\lambda \leq-1) .\end{cases}
$$


This implies that $\varphi(x, y)$ is upper semi-continuous related to the variant $x$. From Corollary 3.2, there exists $\bar{x} \in X$ such that $\phi(\bar{x}, y) \geq 0$ for all $y \in X$.

Since $\varphi(x, y) \leq 0$ for all $x, y \in X$, we know that $\phi(\bar{x}, y)=0$ for all $y \in X$, i.e., $T(\bar{x})=\emptyset$, which is a contradiction.

Now we suppose that the condition (ii) is satisfied. Let $H(x)=T^{-1}(x)=\{y \in X ; x \in T$ (y)\} for $x \in X$. Then $H$ satisfies condition (i). Hence, $H$ has a fixed point in $X$ and so $T$ has a fixed point in $X$. This completes the proof.

Theorem 5.2. Browder type fixed point theorem (Theorem 5.1) is equivalent to Ky Fan's minimax inequality (Corollary 3.2).

Proof. Using the Ky Fan's minimax inequality to prove the Browder fixed point theorem has been shown by Theorem 5.1. Now we prove that Ky Fan's minimax inequality using Browder fixed point theorem. In fact, if the conclusion of Corollary 3.2 is not true, then for each $x \in X$, there exists $y \in X$ such that $f(y)+\varphi(x, y)<f(x)$. Set

$$
T(x)=\{y \in X ; f(y)+\phi(x, y)<f(x)\} .
$$

Then $T(x)$ is a nonempty $F C$-subspace of $X$ for any $x \in X$ and for any $y \in X$.

$$
T^{-1}(y)=\{x \in X ; f(y)+\phi(x, y)<f(x)\}=\{x \in X ; f(x)-\phi(x, y)>f(y)\}
$$

is an open subset of $X$. Therefore there exists $\bar{x} \in X$ such that $\bar{x} \in T(\bar{x})$, i.e., $f(\bar{x})+\phi(\bar{x}, \bar{x})<f(\bar{x})$ and so $\phi(\bar{x}, \bar{x})<0$. This is a contraction. The proof is completed.

\section{Competing interests}

The author declares that they have no competing interests.

Received: 11 October 2011 Accepted: 29 February 2012 Published: 29 February 2012

\section{References}

1. Gwinner, J: On some fixed points and variational inequalities-a circular tour. Nonlinear Anal. 5, 505-583 (1981)

2. Fan, Ky: A minimax inequality and applications. In: O. Shisha (ed.) Inequalities, vol. III, pp. 103-113. Academic Press, New York, London (1972)

3. Shih-sen, Chang, Ying, Zhang: Generalized KKM theorem and variational inequalities. J Math Anal Appl. 159, 10-25 (1991)

4. Tian, GQ: Generalizations of the KKM theorem and the Ky Fan mini-max inequality, with applications to maxmal elements, price equilibrium, and complementarily. J Math Anal Appl. 170, 457-471 (1992). doi:10.1016/0022-247X(92) 90030- $\mathrm{H}$

5. Congjun, Zhang: Existence of solutions of two abstract variational inequalities. Fixed Point Theory Appl. 2, 153-161 (2001)

6. Congjun, Zhang: On existence for the solutions of generalized bi-quasi-variational inequalities and generalized quasivariational. Methematica Applicate. 16, 112-117 (2003)

7. Huang, JH: The matching theorems and coincidence theorems for generalized R-KKM mapping in topological spaces. J Math Anal Appl. 312, 374-382 (2005). doi:10.1016/j.jmaa.2005.03.040

8. Horvath, C: Some results on multi-valued mappings and inequalities without convexity. In: Lin, BL, Simons, S (eds.) Nonlinear and Convex, Lecture notes in pure and Appl Math, vol. 106, pp. 99-106. Dekker, New York (1987)

9. Ding, XP: Himmelberg type fixed point theorem in locally FC-spaces. J Sichuan Normal Univ: Natur Sci. 28, 127-130 (2005)

10. Ding, XP: Generalizations of Himmelberg type fixed point theorem in locally FC-spaces. J Sichuan Normal Univ: Natur Sci. 29, 1-6 (2006)

11. Deng, L, Xia, X: Generalized R-KKM theorem in topological space and their applications. J Math Anal Appl. 285, 679-690 (2003). doi:10.1016/S0022-247X(03)00466-9

12. Browder, F: The fixed point theory of multi-valued mappings in topo-logical vector spaces. Math Ann. 177, 183-301 (1968)

13. Shin, MH, Tan, KK: Generalized quasi-variational inequalities in locally convex topological vector spaces. J Math Anal Appl. 108, 333-343 (1995)

doi:10.1186/1029-242X-2012-49

Cite this article as: Wang: The existence of solutions for general variational inequality and applications in $F C$ spaces. Journal of Inequalities and Applications 2012 2012:49. 